\title{
Psychogenic Movement Disorders
}

Francesca Morgante, MD, PhD; Mark J. Edwards, MD, MBBS, PhD; Alberto J. Espay, MD, MSc, FAAN

\begin{abstract}
Purpose of Review: This review describes the main clinical features of psychogenic (functional) movement disorders and reports recent advances in diagnosis, pathophysiology, and treatment.

Recent Findings: The terminology and definition of patients with psychogenic movement disorders remain subjects of controversy; the term "functional" has been used more frequently in the literature in recent years regarding the neurobiological substrate underpinning these disorders. Correct diagnosis of psychogenic movement disorders should rely not on the exclusion of organic disorders or the sole presence of psychological factors but on the observation or elicitation of clinical features related to the specific movement disorder (ie, a positive or inclusionary rather than exclusionary diagnosis). Sudden onset, spontaneous remissions, and variability over time or during clinical examination are useful "red flags" suggestive of a psychogenic movement disorder. Imaging studies have demonstrated impaired connectivity between limbic and motor areas involved in movement programming and hypoactivity of a brain region that compares expected data with actual sensory data occurring during voluntary movement. Treatment of psychogenic movement disorders begins with ensuring the patient's acceptance of the diagnosis during the initial debriefing and includes nonpharmacologic (cognitive-behavioral therapy, physiotherapy) and pharmacologic options.

Summary: Psychogenic movement disorders represent a challenging disorder for neurologists to diagnose and treat. Recent advances have increased understanding of the neurobiological mechanism of psychogenic movement disorders. Treatment with cognitive strategies and physical rehabilitation can benefit some patients. As short duration of disease correlates with better prognosis, early diagnosis and initiation of treatment are critical.
\end{abstract}

Continuum (Minneap Minn) 2013;19(5):1383-1396.

\section{INTRODUCTION}

Physicians frequently encounter patients with physical symptoms judged to be driven primarily by psychological factors. These patients-variously described as "hysterical," "psychogenic," "functional," "nonorganic," or experiencing "medically unexplained symptoms"- probably represent an underestimated group in a neurology practice: up to $30 \%$ of patients attending neurology outpatient clinics have symptoms that are not at all or only partially explained by organic illness. ${ }^{1}$ Among them, patients presenting with a movement disorder are common, accounting for over half of those with psychogenic motor symptoms. ${ }^{2}$ Their levels of disability and impairment in quality of life are comparable to those of patients with Parkinson disease, ${ }^{3}$ and associated health care costs are high. ${ }^{4}$ Psychogenic (functional) movement disorders represent a diagnostic
Address correspondence to Dr Francesca Morgante, Movement Disorders Unit, Department of Neuroscience, University of Messina, Via Consolare Valeria 1, 98125

Messina, Italy,

fmorgante@gmail.com.

Relationship Disclosure: Dr Morgante serves on a scientific advisory board for Allergan; has received research funding from the Neureca Foundation and honoraria for speaking engagements from Lundbeck,

Novartis, Medtronic, Chiesi

Farmaceutici, and UCB

Pharma; and serves on the

editorial advisory board of

Frontiers in Movement Disorders.

Dr Edwards is supported by grants from the National Institutes of Health Research, Parkinson's UK, UK Dystonia Society, and the Bachmann Strauss Foundation.

Dr Edwards receives royalties

from his book Oxford

Specialist Handbook of

Parkinson Disease and Other

Movement Disorders, serves on

the editorial board of Movement

Disorders Journal, and has

received honoraria from the

Movement Disorders Society and

UCB Pharma. Dr Espay is supported by the K23 career development award (NIMH, 1K23MH092735) and has received grant support from CleveMed/Great Lakes

Neurotechnologies and the Michael J Fox Foundation; personal compensation as a consultant/scientific advisory board member for Solvay (now Abbvie), Chelsea Therapeutics, TEVA, Impax, Merz, Solstice Neurosciences, Eli Lilly, and US WorldMeds; royalties from Lippincott

Williams \& Wilkins and

Cambridge; and honoraria from Novartis, UCB, TEVA, the American Academy of Neurology, and the Movement Disorders Society.

Dr Espay serves as associate editor of Movement Disorders and Frontiers in Movement Disorders and on the editorial board of The European Neurological Journal.

Unlabeled Use of

Products/Investigational Use Disclosure: Drs Morgante, Edwards, and Espay report no disclosures.

(C) 2013, American Academy of Neurology. 


\section{CONTINUUM Psychogenic Movement Disorders}

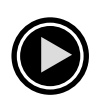

Supplemental digital content: Videos accompanying this article are cited in the text as Supplemental Digital Content. Videos may be accessed by clicking on links provided in the HTML, PDF, and iPad versions of this article; the URLs are provided in the print version. Video legends begin on page 1393 .

\section{KEY POINTS}

- The disability and impairment in quality of life of patients with psychogenic movement disorders may reach magnitudes similar to neurodegenerative diseases, such as Parkinson disease.

- The term "functional" has been proposed to replace the term "psychogenic" when referring to patients with movement disorders that are inconsistent and incongruent with known organic disorders.

Psychogenic tremor attenuates or disappears with distraction and increases with attention; it is of about equal magnitude at rest, during posture holding, and during action. and management challenge for neurologists. This review illustrates the main phenomenologic features that can assist the clinician in formulating an early diagnosis and reviews recent advances in pathophysiology, which have provided a neurobiological basis for these disorders. Finally, this review highlights therapeutic strategies that may benefit some patients.

\section{DEFINITION AND PHENOMENOLOGY}

Psychogenic movement disorders are part of the spectrum of functional neurologic disorders. The definition itself is controversial, as the movements are held as the result of psychological or psychiatric rather than neurologic disturbance. ${ }^{5}$ However, psychopathology is not always evident, and the diagnosis is often made in the absence of any overt psychological dysfunction. The term "functional movement disorders" has been proposed as an alternative. ${ }^{6}$ This term lacks the negative connotation or pejorative impact of "psychogenic," thus favoring acceptance at the time of diagnostic debriefing.

Psychogenic movement disorders account for $1.5 \%$ of all patients seen in neurology clinics, $17 \%$ of all neurologic psychogenic disorders, and $2 \%$ to $20 \%$ of patients referred to movement disorder outpatient clinics, depending on the base population from which these studies were drawn. ${ }^{6}$ Women are more frequently affected than men. Mean age of onset is 37 to 50 years in different studies, although psychogenic movement disorders may manifest in children ${ }^{7}$ and in the elderly. ${ }^{8}$ Diagnosis of psychogenic movement disorders is a multistep process (Figure 9-1) that should be based on specific aspects of history and examination rather than on excluding organic disorders. ${ }^{6}$ Throughout this process, it is important to be aware of the historical and phenomenologic features common to all psychogenic movement disorders (Table 9-1), as well of specific clinical features related to individual movement disorders.

Tremor and dystonia are the most common presentations of psychogenic movement disorders, with parkinsonism, tics, and chorea being the least common. ${ }^{9}$ In contrast to most organic forms of tremor, psychogenic tremor is disrupted or abolished with distracting maneuvers and tends to be of equal magnitude at rest, during maintenance of posture, and while performing an action. Moreover, it may increase in amplitude upon weighting of the limb, entrain to a different examinergiven tapping frequency, and suppress during contralateral ballistic movements ${ }^{10}$ (Supplemental Digital Content 9-1, links.lww.com/CONT/A101).

Psychogenic dystonia is typically fixed, ${ }^{11}$ more frequently affecting the lower than the upper limbs and often developing rapidly after a minor peripheral injury. In some cases, there may be associated pain and dysautonomic features from immobility identical to that seen in reflex sympathetic dystrophy or complex regional pain syndrome type I (a combination sometimes referred to as causalgia-dystonia) $)^{12}$ (Supplemental Digital Content 9-2, links.lww.com/CONT/A102). These features are unlike those of organic dystonia, where abnormal postures (rarely painful) are mobile, being less overt at rest and worse with action and minimized by sensory tricks or "geste antagoniste." 13

Pure psychogenic gait disorders account for $5.7 \%$ of patients with psychogenic movement disorders, although gait abnormalities combined with other movement disorders are more common (Case 9-1), being described in about $40 \%$ of patients. ${ }^{14}$ 


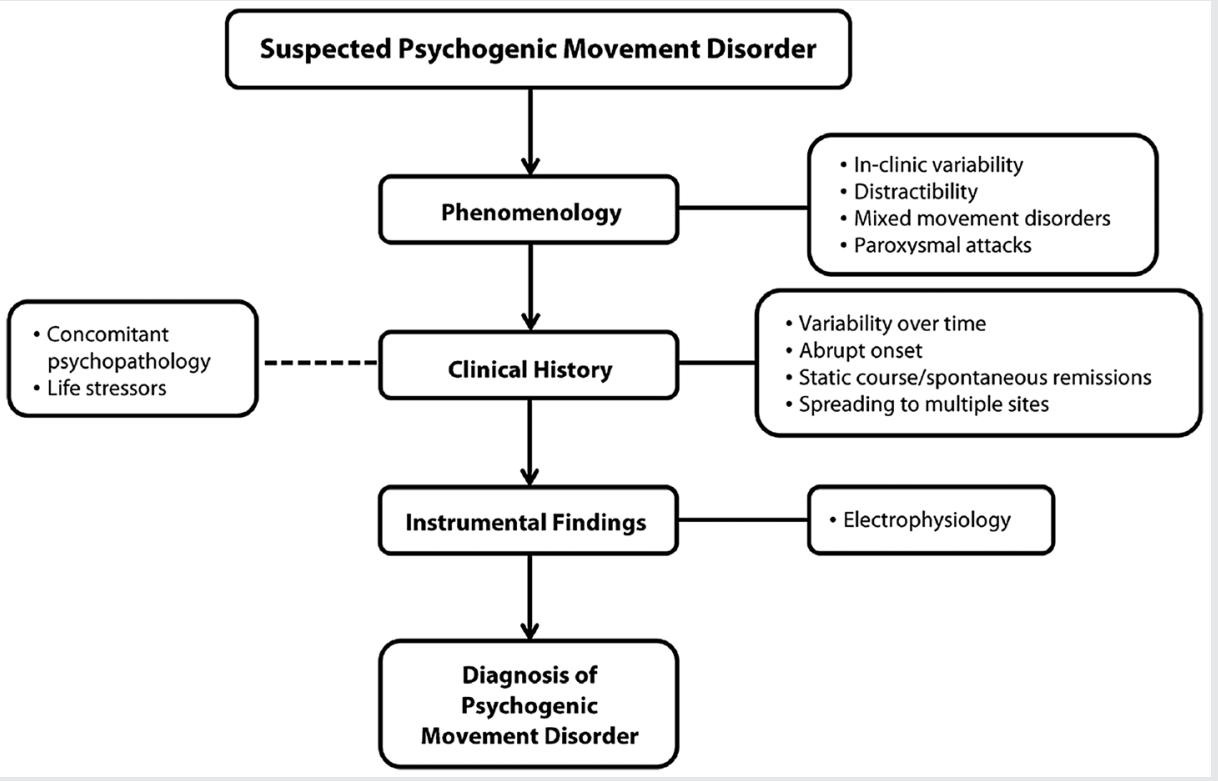

FIGURE 9-1 A proposed algorithm for the diagnosis of psychogenic movement disorders. Diagnosis of psychogenic movement disorder is a multistep process that should encompass demonstration of suggestive phenomenologic features, integration with historical data, and support of electrophysiologic testing. Psychopathology (dotted line) should be carefully searched, although it might not always be evident to a neurologist's evaluation.

Psychogenic movement disorders may affect any topographical distribution, including the craniofacial region $^{15}$ (Case 9-2), palate, ${ }^{16,17}$ neck (posttraumatic painful torticollis), trunk, ${ }^{18}$ and upper and lower limbs. Similarly to young-onset inherited and late-onset idiopathic dystonia, ${ }^{19}$ psychogenic movement disorders can spread from one anatomic site to another, not necessarily following adjacent anatomic segments. ${ }^{15}$ In addition, spontaneous remissions can occur, and relapses may occur in different body parts than those originally affected and with different phenomenologic features. This historical and in-clinic variability represents a distinct feature of psychogenic movement disorders: inconsistency. ${ }^{5}$ Inconsistency can be documented through a reduction or resolution of the psychogenic movement disorder with distraction and worsening when attention is focused on the symptom (particularly in the case of tremor). ${ }^{20}$ Sensitivity to distraction maneuvers (finger tapping movements at different speed, side-to-side tongue movements, counting serial-seven backward, and contralateral rhythmic ballistic movements) can be enhanced by a standardized battery of electrophysiologic tests that demonstrate entrainment, suppression, or pathologic frequency shift in case of psychogenic tremor ${ }^{10}$ and the presence of a premovement potential in cases of psychogenic myoclonus. ${ }^{18}$ Commonly, patients with psychogenic movement disorders present a combination of several types of movement disorders, producing a bizarre clinical picture that is incongruent with any organic disorder (Supplemental Digital Content 9-5, links.lww.com/ CONT/A105). ${ }^{5}$ However, it should be kept in mind that organic disorders may have bizarre presentations. ${ }^{21}$

\section{KEY POINTS}

- Functional dystonia presents as a fixed posturing of the lower limb. Minor peripheral injury often precedes the onset, and pain and dysautonomia tend to complicate the clinical picture, prompting the diagnostic consideration of reflex sympathetic dystrophy (complex regional pain syndrome type I).

Gait abnormalities are frequent in patients with psychogenic movement disorders. Knee buckling and astasia-abasia are the most common patterns in isolated psychogenic gait disorder. Excessive slowness of gait is the most common pattern when other movement disorders are also present.

Clinical symptom variability over the disease course and during clinical examination is one of the major phenomenologic features of psychogenic movement disorders. 


\section{CONTINUUM Psychogenic Movement Disorders}

\section{KEY POINT}

- Abrupt onset and static or fluctuating course with spontaneous remissions are historical clues suggestive of a psychogenic movement disorder.

\section{TABLE 9-1 Historical and Clinical Clues Suggesting a Psychogenic (Functional) Movement Disorder}

- Historical Clues

Abrupt onset

Static course

Spontaneous remissions

Spreading to multiple sites

Paroxysmal symptoms

Psychiatric comorbidities

Secondary gain

Risk factors for conversion disorder

Psychological stressors

Mild physical trauma shortly before onset

Multiple somatizations/undiagnosed conditions

Previous exposure to a disease model

Variability of phenomenology and severity over time

\section{- Clinical Clues}

Inconsistency

Increase with attention, decrease with distraction

Entrainment of tremor to the frequency of repetitive movements

Variability of phenomenology during examination

Selective disability or functional disability out of proportion to examination findings

Incongruency

Mixed movement disorders

Atypical stimulus sensitivity

Paroxysmal attacks

False weakness

False sensory signs

Deliberate slowness

Suggestibility

a Modified with permission from Gupta A, Lang AE, Curr Op Neurol. ${ }^{5}$ @) 2009 Lippincott Williams \& Wilkins, Inc. journals.Iww. com/co-neurology/pages/articleviewer.aspx?year=2009\&issue= 08000\&article $=00015 \&$ type $=$ abstract.

Apart from inconsistency and incongruency, ${ }^{22}$ several other clues from clinical history and examination are helpful in diagnosing psychogenic movement disorders ${ }^{5}$ (see Table 9-1). Symptom onset is often abrupt, and severity tends to be maximal at the outset; symptoms may follow a minor injury, disproportionate to the initial triggering factor. In terms of disease progression, patients with psychogenic movement disorders can display either a static or a fluctuating course with spontaneous remissions, sudden relapses, and changing phenomenology over time. ${ }^{6}$ During examination, it may be helpful to assess response (nonphysiologic amelioration or worsening) 


\section{Case 9-1}

A 51-year-old man exhibited gait impairment, right hand action tremor, and left leg resting tremor of sudden onset after a spearfishing accident 8 years ago. After 4 years, fixed dystonia appeared in his left upper limbs and later in the right upper limb, associated with excessive slowness of movement. Neurologic examination showed brief motor arrests during gait initiation and turning, steppage of the left foot, and fixed posture of both hands. He was unable to run. During examination while sitting, fixed hand posturing disappeared during distraction maneuvers (Supplemental Digital Content 9-3, links./ww.com/CONT/A103). His leg tremor was present when the foot was resting on the anterior sole but not when resting on the heel or when unsupported. Deliberate slowness in both upper limbs was demonstrated during finger-to-nose movements and repetitive arm movements, although spontaneous movements were normal, as was writing. Psychiatric evaluation showed mild depression. He was treated with escitalopram without improvement of involuntary movements. He was diagnosed to have psychogenic tremor and psychogenic gait disorder but declined to undergo psychotherapy.

Comment. This man received a psychogenic movement disorder diagnosis many years after symptom onset. Diagnosis was supported by phenomenologic features (clinical variability over time and during examination, suppression of symptoms with distraction, increase with attention, mixed movement disorders) in the absence of overt psychological abnormalities.

\section{Case 9-2}

A 35-year-old woman was evaluated for left hemifacial spasm, which started 4 years before. She had been treated with botulinum toxin type $A$ several times without benefit and had twice undergone microvascular decompression, which resolved her symptoms for only 2 days after the first surgical procedure. Neurologic examination demonstrated reduction of left-side facial spasm during distracting maneuvers (eg, moving the tongue from side to side) (Supplemental Digital Content 9-4, links.IWw.com/CONTIA104). Contraction of frontalis muscle involved the eyebrow contralateral to the spasm of the orbicularis oculi. Electrophysiologic evaluation disclosed a normal blink reflex without evidence of ephaptic transmission and disappearance of EMG contraction during sleep, by polysomnography. Psychiatric evaluation led to the diagnosis of conversion disorder. Partial relief of symptoms was achieved with cognitive-behavioral therapy.

Comment. This woman received a diagnosis of psychogenic facial spasm. Several phenomenologic features, such as contraction of frontalis muscle contralateral to the spasm of the orbicularis oculi and electrophysiologic testing, supported the diagnosis. 


\section{CONTINUUM Psychogenic Movement Disorders}

\section{KEY POINTS}

Diagnosis of

psychogenic movement disorders should rely on positive features.

Electrophysiology is useful for documenting laboratory-supported (definite) psychogenic tremor and psychogenic myoclonus.

- Feigning (malingering) is exceedingly rare as a source of psychogenic movement disorders.

The most common underlying psychopathology is conversion disorder. to selected interventions, such as somatic trigger points and vibrating tuning fork. Additional neurologic signs can suggest a psychogenic movement disorder, ${ }^{5}$ such as excessive startle response to a stimulus, nonanatomic sensory loss or spread of movement, give-away weakness, presence of convergence spasms, ${ }^{23}$ and abduction finger sign (if upper limb paresis co-occurs). ${ }^{24}$

\section{DIAGNOSIS}

Diagnosis of a psychogenic movement disorder should take into consideration the above-mentioned red flags from history and clinical examination as well as selected electrophysiologic findings. Neither the absence nor the presence of psychological or psychiatric disturbances per se should influence the diagnosis, as they may not be present ${ }^{25}$ and many basal ganglia disorders have psychiatric symptoms and movement disorders that may be bizarre or difficult to categorize (eg, Huntington disease, chorea-acanthocytosis, young-onset Parkinson disease). Diagnosis can be complicated by the coexistence of psychogenic movement disorders with organic movement disorders. ${ }^{26}$

Three sets of clinical criteria-the Fahn-Williams, ${ }^{22}$ the Shill-Gerber, ${ }^{27}$ and the Gupta-Lang ${ }^{5}$ criteria-have been developed to formalize and assist in the diagnostic process. The Fahn-Williams criteria specify levels of diagnostic certainty (possible, probable, clinically definite) based on incongruence or inconsistency of the movement disorder, with response to suggestion, placebo, or psychotherapy defining the highest level of diagnostic certainty. ${ }^{22}$ The Shill-Gerber criteria also rely on clinical elements that suggest inconsistency with an organic illness, but the level of diagnostic certainty is primarily based on nonphenomenologic factors, such as disproportionate pain or fatigue, sec- ondary gain, and exposure to a disease model. ${ }^{27}$ Although the Fahn-Williams and Shill-Gerber criteria aim to deliver a diagnosis based on positive rather than exclusionary criteria, they show poor inter-rater reliability when applied to cases that featured a degree of clinical uncertainty in the diagnosis. ${ }^{28}$ This failure may be related to a disproportionately larger value assigned to psychological than phenomenologic features (particularly in the Shill-Gerber criteria $^{29}$ and a lack of reliance on electrophysiologic testing. The GuptaLang criteria suggested a "laboratorysupported, definite" diagnostic category based on electrophysiologic findings (Table 9-2), such as frequency analysis for psychogenic tremor and EMG-EEG back-averaging for psychogenic myoclonus. ${ }^{18}$ Specifically, a combination of electrophysiologic tests has recently been proposed to differentiate psychogenic from organic tremor ${ }^{10}$; these tests include entrainment or frequency changes with tapping (Figure 9-2).

\section{PATHOPHYSIOLOGY}

Two areas present significant difficulty in understanding how psychogenic movement disorder symptoms are generated. The first is that the clinical signs presented by patients look identical to movements voluntarily produced or feigned, which has led to the misperception that malingering is the mechanism behind most patients with psychogenic movement disorders. ${ }^{30,31}$ In fact, only a small proportion of patients with psychogenic movement disorders are involved in litigation, ${ }^{32}$ and general consensus is that only a small number are malingering. Furthermore, functional imaging studies have shown group differences in patterns of brain activation when patients with weakness and healthy volunteers feigning weakness are asked to try to move their weak leg. ${ }^{33}$ 


\section{TABLE 9-2 Electrophysiologic Studies Used During the Evaluation of Selected Patients With Psychogenic Movement Disorders}

\section{- Electrophysiologic Studies in Psychogenic Movement Disorders}

Nerve conduction studies, EMG, and blink reflex (only abnormal in peripheral nerve lesions and ephaptic transmission-eg, hemifacial spasm)

Polygraphic surface EMG recordings

Accelerometry

EMG-EEG back averaging with Bereitschaftspotential (premovement cortical potential) study

\section{- Electrophysiologic Findings in Psychogenic Tremor}

Changes in frequency and amplitude during distractibility maneuvers

Entrainment, suppression, or frequency shift during contralateral finger tapping

Incorrect tapping performance

Increase with loading

Coactivation sign

Pause of tremor during contralateral ballistic movements
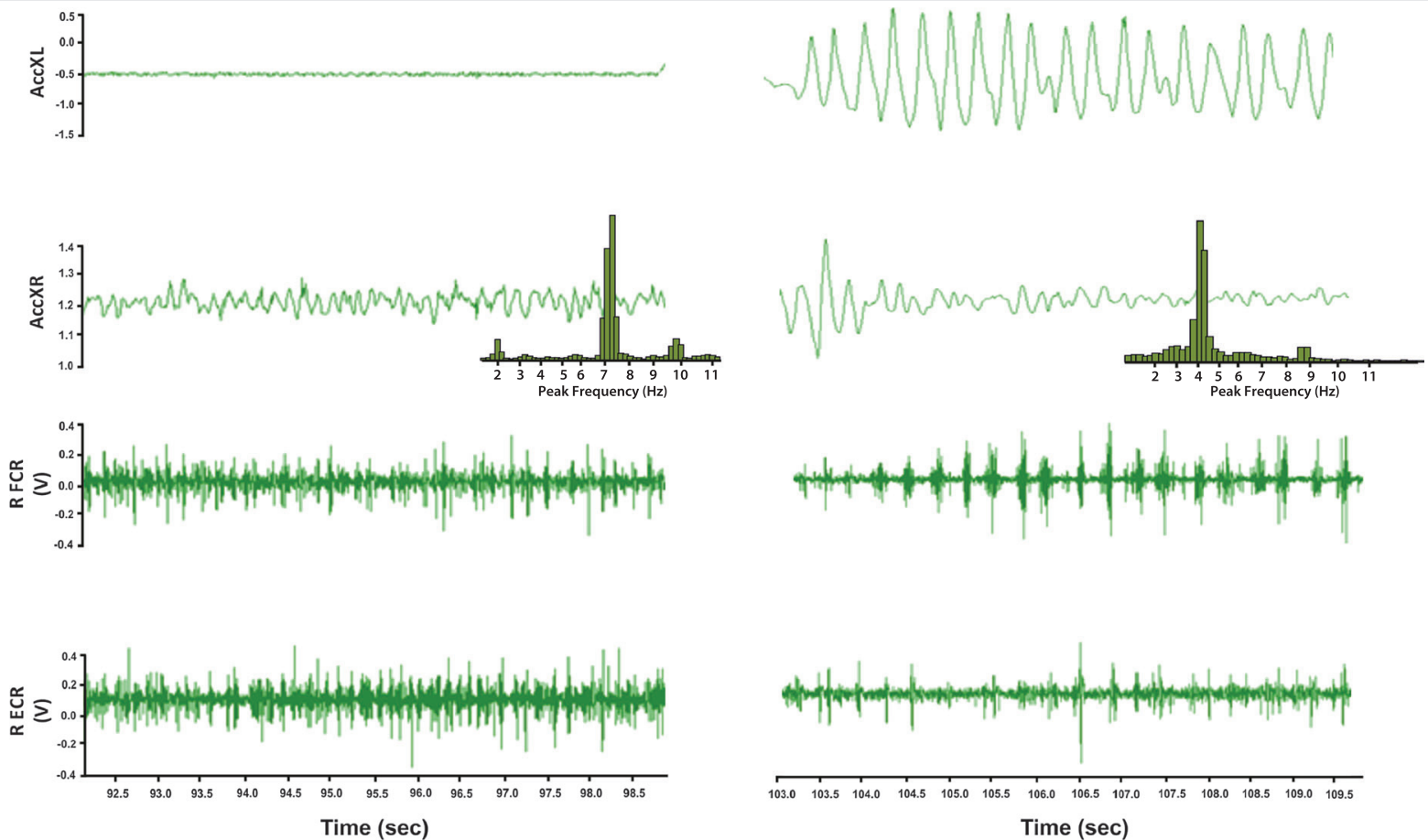

FICURE 9-2 Electrophysiologic demonstration of tremor entrainment in a patient with psychogenic tremor. Accelerometry recordings from an accelerometer attached to the hand affected by tremor (AccXR) and to the unaffected hand (AcCXL). Below are EMG recordings from the affected hand (RFCR = right flexor carpi radialis; RECR = right extensor carpi radialis). Tremor frequency analysis by fast Fourier transformer shows a $7-\mathrm{Hz}$ peak frequency of tremor (from 92.5 to $98.5 \mathrm{~ms}$ ); finger tapping of unaffected hand at $4 \mathrm{~Hz}$ entrained the affected hand at the same frequency (from 103 to $109.5 \mathrm{~ms})$. 


\section{CONTINUUM Psychogenic Movement Disorders}

\section{KEY POINT}

- Patients with psychogenic movement disorders demonstrate heightened attention toward the affected body part, exaggerated perception of symptom duration over the course of a day, and distorted expectations and beliefs about symptoms.
The second area of difficulty is related to the absence of overt psychopathology. Counseling regarding "stress-induced" symptoms may not be acceptable to a large number of patients who may not report clear psychological stressors, an essential criterion for the diagnosis of conversion disorder in the Diagnostic and Statistical Manual of Mental Disorders, Fourth Edition (DSM-IV). ${ }^{25}$

As highlighted above, correct diagnosis of psychogenic movement disorders should rely not on the presence of psychological factors but on positive features related to the movement disorder. These features reflect (1) symptoms that require attention to manifest and (2) mismatch between the patient's beliefs/expectations and real sensory data. Indeed, in the latest revision to the DSM (DSM-V), the need for a psychological stressor has been removed as well as the need to exclude malingering or factitious disorder.

Pathophysiologic research has therefore focused on demonstrating experimentally that the explicit aspects of movement control are affected in patients with psychogenic movement disorders $^{34}$ and that visual attention toward movement is a common feature of patients. ${ }^{20}$ Another study employing tremor assessment by actigraphy and patient-based diaries demonstrated that patients with psychogenic tremor overestimated the amount of the day they experienced tremor to a significantly greater extent than patients with organic tremor. ${ }^{35}$ As a group they had tremor on average for 30 minutes a day, and reported it being present for $80 \%$ to $90 \%$ of the time. A preliminary study looking at decision-making styles in patients with psychogenic movement disorders showed a "jumping to conclusions" pattern of decision making, which is also seen in patients with delusions. ${ }^{36}$ This provides a potentially interesting link between the neurobiological understanding of delusions and that of psychogenic movement disorders and other psychogenic symptoms.

The mismatch between sensory data and expectations/predictions about these data has been also highlighted by functional imaging studies. In psychogenic tremor, reduced activity has been found in the temporoparietal junction ${ }^{37}$ only when patients were scanned during their habitual tremor and not when they deliberately mimicked their tremor; the temporoparietal junction is a key node in the network that informs expectations and predictions based on sensory data. Such temporoparietal junction hypoactivity may reflect the lack of an appropriate sensory prediction signal typically associated with voluntary movements and could explain why psychogenic movements, while looking voluntarily generated, are not experienced as such by patients. ${ }^{37}$ This is in keeping with a behavioral study showing a lack of the normal perception of intention to move before a voluntary movement in patients with psychogenic tremor ${ }^{38}$ and may contribute to the impaired somatosensory processing in patients with psychogenic dystonia. ${ }^{39}$

Functional imaging studies have demonstrated abnormal connectivity of motor areas involved in motor preparation and limbic areas during performance of a motor act or in response to an emotional stimulus. ${ }^{40,41}$ During movement preparation, patients with psychogenic tremor exhibit low activation of the supplementary motor area involved in motor initiation and inhibition, and high activation of limbic areas, implicated in emotional processing. ${ }^{41}$ During performance of internally generated movements, there is lower connectivity between supplementary motor 
area and dorsolateral prefrontal cortices but higher connectivity between the supplementary motor area and the amygdala (an important node in the limbic system) when exposed to emotional stimuli, compared with controls. ${ }^{40}$

Regarding the question of how behavioral, imaging, and electrophysiologic findings can be incorporated in a unitary model to explain the production of psychogenic movement disorders, a growing body of literature supports the hypothesis that generation of an abnormal belief/expectation relating to the symptoms is a crucial step. ${ }^{41-43}$ The appearance of this belief/expectation could arise from a number of factors, including emotionally traumatic events or physical triggers (injury, organic illness). The second step required for symptom generation is the unwanted activation of an abnormal motor pattern related to the symptom $^{41,43}$; it is proposed that a hypoactive voluntary action selection system can be diverted to previously learned motor routines, which can be activated by emotional contextual cues. ${ }^{41}$ Here a role may exist for abnormal limbic system activation and self-directed attention. Activation of this "conversion motor representation" circumvents the patient's sense of agency for the movement (agency applies to the experience that one is in control of one's actions), and thus it is misattributed by the patient as being involuntary and out of his or her volitional control.

Considerable work remains to be done to fully understand the pathophysiology of these intriguing symptoms. This work may not only lead to insights about patients with psychogenic movement disorders but also about the overall generation of movements by the human brain.

\section{TREATMENT}

Treatment of psychogenic movement disorders is a process that starts from diagnostic debriefing, passes through acceptance of diagnosis, and leads to multiple strategies, including nonpharmacologic and pharmacologic options (Table 9-3). Unfortunately, current treatments are based on single case reports or small cohort studies. Early initiation of treatment increases the rate of sustained remission.

\section{Diagnostic Debriefing}

The most important element in the management of clinically definite

\section{KEY POINT}

Functional imaging studies suggest abnormal coupling between limbic (emotional) and higher motor areas as well as hypoactivity of the temporoparietal junction, which impairs expectations and predictions of movement based on sensory data. These findings may also explain the lack of agency (sense of self-control) over abnormal movements reported by patients with psychogenic movement disorders.

\title{
TABLE 9-3 Pharmacologic and Nonpharmacologic Interventions Used in Selected Psychogenic Movement Disorders
}

\author{
- Pharmacologic Intervention \\ Antidepressants \\ - Nonpharmacologic Interventions \\ Cognitive-behavioral therapy \\ Psychodynamic psychotherapy \\ Physical and occupational therapy \\ Physical activity \\ Transcutaneous electrical stimulation \\ Acupuncture \\ Repetitive transcranial magnetic stimulation
}




\section{CONTINUUM Psychogenic Movement Disorders}

\section{KEY POINTS \\ - Empathetic and disability-validating debriefing of the diagnosis is critical to ensure patients embrace the diagnosis and actively participate in their treatment program. \\ - The diagnosing neurologist is best suited to coordinate multidisciplinary care and assume follow-up care to reexamine and reassure the patient and to simplify pharmacotherapy. \\ - Cognitive-behavioral therapy is emerging as an intervention that may yield long-term remission in patients with psychogenic movement disorders by shifting presumably pathogenic dysfunctional schemas (cognitive distortions) into functional, adaptive core beliefs.}

psychogenic movement disorders is the disclosure of the diagnosis to patients in a manner that is empathetic and nonjudgmental, and validating of the disability accrued. ${ }^{44}$ Only such an approach can foster the necessary acceptance of the diagnosis, which is the strongest predictor of favorable outcome after psychotherapy. ${ }^{45}$ For the vast majority of patients with conversion disorder or, less commonly, somatization disorder, three critical points are worth emphasizing during the diagnostic debriefing: (1) that the movement abnormalities are not deliberate but are subconsciously generated (eg, "you are not making this up"); (2) that these do not mean psychiatric disease (eg, "you are not crazy"); and (3) that the movements are real and preventing normal function (eg, "you are disabled").

Leaving no margin of doubt as to the diagnosis justifies the recommendation, also stated to the patient once the diagnosis is established, that no further standard neurologic investigations are warranted. If the three reasons above are explicitly stated, the decision to withhold testing will not be viewed as dismissive or callous, and will prevent unnecessary use of costly resources. The accuracy of the diagnosis (and the prospects for subsequent efforts at reversing symptoms) can be greatly helped by disclosing to patients how the diagnosis is made, including elicitation of their physical signs such as tremor retrainment. ${ }^{46}$

The goals of the diagnostic debriefing are, ultimately, to ensure that the patient walks away with (1) a validation of the motor disability, (2) a decisive acceptance of the diagnosis (and thus avoiding the doctor-shopping pitfall that comes from the perception that "no one has told me what is wrong with me"), (3) a sense of partnership with the neurologist, and (4) an under- standing that individually tailored multidisciplinary treatment, which should include psychological intervention, is often appropriate.

\section{Nonpharmacologic Approaches}

Neurologists are not only uniquely trained to establish the diagnosis of psychogenic movement disorders (psychiatrists or physiatrists cannot) but also specially positioned to provide periodic follow-up (reexamining, reassuring the patient about the absence of structural disease, and simplifying pharmacotherapy, among others) and coordination of care. On a case-by-case basis, patients can derive benefits with the collaboration of a clinical psychologist, physical therapist, and/or psychiatrist, all of whom should be informed regarding the nature of psychogenic movement disorders and the goals of therapy. An outpatient, 1-week, intensive goal-oriented physiotherapy program conducted at the Mayo Clinic, based on the concept of motor reprogramming with minimal overtly psychological input, has yielded a 60\% rate of success (marked improvement or remission) in 2-year outcomes compared to treatment as usual (in an historical control group). ${ }^{47}$ Standardized physical activity has been also suggested: three sessions per week of structured lowmoderate intensity walking has been shown to be beneficial, determining an improvement of about $70 \%$ of the Psychogenic Movement Disorder Rating Scale. ${ }^{48}$

Cognitive-behavioral therapy is a promising approach to the management of psychogenic movement disorders, and efforts are ongoing to delve into its efficacy in a systematic fashion. ${ }^{49}$ Cognitive-behavioral therapy aims at instructing patients to identify and ultimately disrupt the behavioral, physiologic, and cognitive responses experienced with stress, which often are at the root of psychogenic behaviors. 
Through "catching" automatic, often negative thoughts and linking them to their associated emotions and behaviors, patients can then be helped to trace those negative thoughts back to deeply held cognitive distortions or dysfunctional schemas, which are thought to fuel the maintenance of abnormal psychogenic behaviors. Cognitivebehavioral therapy is particularly helpful in situations when low self-esteem, depression, and anxiety are the main psychopathologic elements.

Psychodynamic psychotherapy, a form of psychoanalytic therapy aimed at reshaping the intrapsychic structure based on the link between historical and early life experiences, parenting dynamics, enduring personality traits, and current life experiences and problematic emotions, has also been shown to be of benefit in a single-blind clinical trial. ${ }^{50}$ In this form of therapy, a treating psychiatrist also uses antidepressant or anxiolytic medications as deemed warranted to effectively treat coexisting anxiety or depression.

Transcranial magnetic stimulation has also been reported to be effective in patients with functional movement disorders, but the exact mechanism is uncertain at this time. It seems most likely, given the parameters of stimulation used and the dramatic effects reported, that this is a placebo effect rather than a neuromodulatory effect due to the transcranial magnetic stimulation itself. ${ }^{51}$

\section{Pharmacologic Options}

Although the abnormal movements themselves should not be targets of pharmacotherapy, associated psychiatric illnesses might. Indeed, when comorbid depression and anxiety are present, treatment of these conditions may expedite recovery. The response to antidepressants, however, depends on the underlying psychopathology. In the setting of conversion disorder, depressed patients with psychogenic movement disorders can exhibit a favorable response to antidepressants. ${ }^{52}$ Patients with somatization disorder, primary hypochondriasis, and factitious disorder or malingering did not benefit in this study.

\section{SUMMARY}

Psychogenic movement disorders are common and disabling. They should be diagnosed where possible on the basis of positive aspects of the history and examination. A developing understanding of the pathophysiology of these disorders has moved away from pure psychological explanations in favor of broader biopsychosocial models. Although prognosis can be poor (associated with delayed diagnosis), treatment with physical and/or cognitive-behavioral approaches can be successful.

\section{VIDEO LEGENDS \\ Supplemental Digital Content 9-1}

Psychogenic tremor. Video shows a 45-year-old woman with sudden-onset right hand tremor. Tremor was present at rest, with variable posturing of right hand, and during finger movements. Tremor was disrupted and its frequency changed during finger tapping with the unaffected left hand at different rates. Tremor was temporarily suppressed when engaged in the finger-nose task with the unaffected hand.

links.lww.com/CONT/A101

(C) 2013 American Academy of Neurology.

\section{Supplemental Digital Content 9-2}

Fixed dystonia. Video shows a 62-year-old woman with sudden-onset left foot posturing after a minor motor vehicle accident 7 years ago. Fixed foot flexion and inversion are present, with excessive pain and associated abnormalities of skin coloration and sweating. She was diagnosed with complex regional pain syndrome type 1 . links.lww.com/CONT/A102

(C) 2013 American Academy of Neurology.

\section{Supplemental Digital Content 9-3}

Psychogenic gait disorder. Video shows the patient described in Case 9-1, a 51-year-old man exhibiting gait impairment, right hand action tremor, and left leg resting tremor. Neurologic examination showed brief motor arrests during

\section{KEY POINT}

Although

pharmacotherapy for

the movements

themselves is

discouraged, treatment

of comorbid depression

and anxiety

may expedite the road

to recovery. 


\section{CONTINUUM Psychogenic Movement Disorders}

gait initiation and turning, steppage of the left foot, and fixed posture of both hands. While sitting, fixed hand posturing disappeared during distraction maneuvers. Leg tremor was present when the foot was resting on the anterior sole but not when resting on the heel or when unsupported. Deliberate slowness in both upper limbs was demonstrated during finger-to-nose movements and repetitive arm movements, although spontaneous movements were normal, as was writing.

links.lww.com/CONT/A103

(c) 2013 American Academy of Neurology.

\section{Supplemental Digital Content 9-4}

Psychogenic facial spasm. Video shows the patient described in Case 9-2, a 35-year-old woman evaluated for left hemifacial spasm. Neurologic examination demonstrated reduction of left side facial spasm during distracting maneuvers (eg, moving the tongue from side to side). Contraction of the frontalis muscle involved the eyebrow contralateral to the spasm of the orbicularis oculi. Electrophysiologic evaluation disclosed a normal blink reflex without evidence of ephaptic transmission and disappearance of EMG contraction during sleep, by polysomnography. Psychiatric evaluation led to the diagnosis of conversion disorder.

links.lww.com/CONT/A104

(C) 2013 American Academy of Neurology.

\section{Supplemental Digital Content 9-5}

Psychogenic paroxysmal generalized dystonia. Video shows a 54-year-old woman with paroxysmal hyperextension of the right upper limb with wrist flexion and involuntary trunk movements. During these episodes, she exhibited lateral trunk deviation to the right side. No clear precipitating factors were evident.

links.lww.com/CONT/A105

(C) 2013 American Academy of Neurology.

\section{REFERENCES}

1. Stone J, Carson A. Movement disorders: psychogenic movement disorders: what do neurologists do? Nat Rev Neurol 2009;5(8): 415-416.

2. Crimlisk HL, Bhatia $K$, Cope $H$, et al. Slater revisited: 6 year follow up study of patients with medically unexplained motor symptoms. BMJ 1998;316(7131):582-586.

3. Anderson KE, Gruber-Baldini AL, Vaughan CG, et al. Impact of psychogenic movement disorders versus Parkinson's on disability, quality of life, and psychopathology. Mov Disord 2007;22(15):2204-2209.
4. Hinson VK, Haren WB. Psychogenic movement disorders. Lancet Neurol 2006;5(8):695-700.

5. Gupta A, Lang AE. Psychogenic movement disorders. Curr Opin Neurol 2009;22(4): 430-436.

6. Edwards MJ, Bhatia KP. Functional (psychogenic) movement disorders: merging mind and brain. Lancet Neurol 2012;11(3):250-260.

7. Canavese C, Ciano C, Zibordi F, et al. Phenomenology of psychogenic movement disorders in children. Mov Disord 2012;27(9): $1153-1157$.

8. Batla A, Stamelou M, Edwards MJ, et al. Functional movement disorders are not uncommon in the elderly. Mov Disord 2013;28(4):540-543.

9. Lang AE. General overview of psychogenic movement disorders: epidemiology, diagnosis, and prognosis. Psychogenic movement disorders - neurology and neuropsychiatry. Philadelphia: Lippincott Williams \& Wilkins, 2006:35-41.

10. Schwingenschuh $P$, Katschnig $P$, Seiler $S$, et al. Moving toward "laboratory-supported" criteria for psychogenic tremor. Mov Disord 2011:26(14):2509-2515.

11. Schrag A, Trimble M, Quinn N, Bhatia K. The syndrome of fixed dystonia: an evaluation of 103 patients. Brain 2004;127(pt 10):2360-2372.

12. Lang $A E$, Chen R. Dystonia in complex regional pain syndrome type I. Ann Neurol 2010;67(3):412-414.

13. Albanese A, Lalli S. Is this dystonia? Mov Disord 2009;24(12):1725-1731.

14. Baik JS, Lang AE. Gait abnormalities in psychogenic movement disorders. Mov Disord 2007;22(3):395-399.

15. Fasano A, Valadas A, Bhatia KP, et al. Psychogenic facial movement disorders: clinical features and associated conditions. Mov Disord 2012;27(12):1544-1551.

16. Stamelou M, Saifee TA, Edwards MJ, Bhatia KP. Psychogenic palatal tremor may be underrecognized: reappraisal of a large series of cases. Mov Disord 2012;27(9):1164-1168.

17. Biller J, Espay AJ. Nosography of the "essential": volitional palatal tremor. Neurology. In press.

18. Esposito M, Edwards MJ, Bhatia KP, et al. Idiopathic spinal myoclonus: a clinical and neurophysiologic assessment of a movement disorder of uncertain origin. Mov Disord 2009;24(16):2344-2349.

19. Martino D, Berardelli A, Abbruzzese $G$, et al Age at onset and symptom spread in primary adult-onset blepharospasm and cervical dystonia. Mov Disord 2012;27(11):1447-1450. 
20. van Poppelen D, Saifee TA, Schwingenschuh $P$, et al. Attention to self in psychogenic tremor. Mov Disord 2011;26(14):2575-2576.

21. Simon DK, Nishino S, Scammell TE. Mistaken diagnosis of psychogenic gait disorder in a man with status cataplecticus ("limp man syndrome"). Mov Disord 2004;19(7): 838-840.

22. Williams DT, Ford B, Fahn S. Phenomenology and psychopathology related to psychogenic movement disorders. Adv Neurol 1995; 65:231-257.

23. Fekete R, Baizabal-Carvallo JF, Ha AD, et al. Convergence spasm in conversion disorders: prevalence in psychogenic and other movement disorders compared with controls. J Neurol Neurosurg Psychiatry 2012;83(2):202-204.

24. Tinazzi $M$, Simonetto $S$, Franco $L$, et al. Abduction finger sign: a new sign to detect unilateral functional paralysis of the upper limb. Mov Disord 2008;23(16):2415-2419.

25. Kranick S, Ekanayake V, Martinez V, et al. Psychopathology and psychogenic movement disorders. Mov Disord 2011;26(10):1844-1850.

26. Ranawaya R, Riley D, Lang A. Psychogenic dyskinesias in patients with organic movement disorders. Mov Disord 1990;5(2):127-133.

27. Shill H, Gerber P. Evaluation of clinical diagnostic criteria for psychogenic movement disorders. Mov Disord 2006;21(8):1163-1168.

28. Morgante F, Edwards MJ, Espay AJ, et al. Diagnostic agreement in patients with psychogenic movement disorders. Mov Disord 2012;27(4):548-552.

29. Voon $V$, Lang $A E$, Hallett M. Diagnosing psychogenic movement disorders - which criteria should be used in clinical practice? Nat Clin Pract Neurol 2007;3(3):134-135.

30. Kanaan R, Armstrong D, Barnes P, Wessely S. In the psychiatrist's chair: how neurologists understand conversion disorder. Brain 2009;132(pt 10):2889-2896.

31. Kanaan RA, Armstrong D, Wessely SC. Neurologists' understanding and management of conversion disorder. J Neurol Neurosurg Psychiatry 2011;82(9):961-966.

32. Carson A, Stone J, Hibberd C, et al. Disability, distress and unemployment in neurology outpatients with symptoms 'unexplained by organic disease'. J Neurol Neurosurg Psychiatry 2011;82(7):810-813.

33. Stone J, Zeman A, Simonotto E, et al. FMRI in patients with motor conversion symptoms and controls with simulated weakness. Psychosom Med 2007;69(9):961-969.
34. Parees I, Kassavetis P, Saifee TA, et al. Failure of explicit movement control in patients with functional motor symptoms. Mov Disord 2013;28(4):517-523.

35. Parees I, Saifee TA, Kassavetis $P$, et al. Believing is perceiving: mismatch between self-report and actigraphy in psychogenic tremor. Brain 2012;135(pt 1):117-123.

36. Parees I, Kassavetis $P$, Saifee TA, et al. 'Jumping to conclusions' bias in functional movement disorders. J Neurol Neurosurg Psychiatry 2012;83(4):460-463.

37. Voon V, Gallea C, Hattori N, et al. The involuntary nature of conversion disorder. Neurology 2010;74(3):223-228.

38. Edwards MJ, Moretto G, Schwingenschuh P, et al. Abnormal sense of intention preceding voluntary movement in patients with psychogenic tremor. Neuropsychologia 2011;49(9):2791-2793.

39. Morgante $F$, Tinazzi M, Squintani G, et al. Abnormal tactile temporal discrimination in psychogenic dystonia. Neurology 2011;77(12): 1191-1197.

40. Voon V, Brezing C, Gallea C, et al. Emotional stimuli and motor conversion disorder. Brain 2010;133(pt 5):1526-1536.

41. Voon V, Brezing C, Gallea C, Hallett M. Aberrant supplementary motor complex and limbic activity during motor preparation in motor conversion disorder. Mov Disord 2011;26(13):2396-2403.

42. Brown RJ. Psychological mechanisms of medically unexplained symptoms: an integrative conceptual model. Psychol Bull 2004;130(5):793-812.

43. Edwards MJ, Adams RA, Brown H, et al. A Bayesian account of 'hysteria'. Brain 2012;135 (pt 11):3495-3512.

44. Clark MR. Psychogenic disorders: a pragmatic approach for formulation and treatment. Semin Neurol 2006;26(3):357-365.

45. Espay AJ, Goldenhar LM, Voon V, et al. Opinions and clinical practices related to diagnosing and managing patients with psychogenic movement disorders: an international survey of movement disorder society members. Mov Disord 2009;24(9): 1366-1374.

46. Stone J, Edwards M. Trick or treat? Showing patients with functional (psychogenic) motor symptoms their physical signs. Neurology 2012;79(3):282-284.

47. Czarnecki K, Thompson JM, Seime R, et al. Functional movement disorders: successful treatment with a physical therapy rehabilitation 


\section{CONTINUUM Psychogenic Movement Disorders}

protocol. Parkinsonism Relat Disord 2012; 18(3):247-251.

48. Dallocchio C, Arbasino C, Klersy C, Marchioni E. The effects of physical activity on psychogenic movement disorders. Mov Disord 2010;25(4): 421-425.

49. LaFrance WC Jr, Friedman JH. Cognitive behavioral therapy for psychogenic movement disorder. Mov Disord 2009;24(12):1856-1857.

50. Hinson VK, Weinstein S, Bernard B, et al. Single-blind clinical trial of psychotherapy for treatment of psychogenic movement disorders. Parkinsonism Relat Disord 2006;12(3):177-180

51. Pollak TA, Nicholson TR, Edwards MJ, David AS. A systematic review of transcranial magnetic stimulation in the treatment of functional (conversion) neurological symptoms. J Neurol Neurosurg Psychiatry. In press.

52. Voon V, Lang AE. Antidepressant treatment outcomes of psychogenic movement disorder. J Clin Psychiatry 2005; 66(12):1529-1534. 